\title{
Adenosine Triphosphate-dependent Transport of Doxorubicin, Daunomycin, and Vinblastine in Human Tissues by a Mechanism Distinct from the P-Glycoprotein
}

\author{
Sanjay Awasthi, * Sharad S. Singhal, * Sanjay K. Srivastava, ${ }^{\star}$ Piotr Zimniak, ${ }^{\star}$ Krishna K. Bajpai, ${ }^{\star}$ Manju Saxena, \\ Rashmi Sharma, ${ }^{\star}$ Stephen A. Ziller III, Eugene P. Frenkel," Shivendra V. Singh, " Nong G. He, ${ }^{\star}$ and Yogesh C. Awasthi ${ }^{*}$ \\ ${ }^{*}$ Department of Internal Medicine, Division of Hematology and Oncology, and ${ }^{\ddagger}$ Department of Human Biological Chemistry and \\ Genetics, University of Texas Medical Branch, Galveston, Texas $77555 ;{ }^{\S}$ Department of Medicine, and Department of Biochemistry \\ and Molecular Biology, University of Arkansas for Medical Sciences, Little Rock, Arkansas 72205; \\ "Department of Internal Medicine, The University of Texas Southwestern Medical Center, Dallas, \\ Texas 75235; and "Mercy Hospital of Pittsburgh, Pittsburgh, Pennsylvania 15219
}

\begin{abstract}
Previous studies have demonstrated that a human glutathione conjugate transporter, designated as dinitrophenyl-S-glutathione ATPase (DNP-SG ATPase), catalyzed ATP hydrolysis in the presence of several amphiphilic compounds other than glutathione conjugates (Singhal, S. S., R. Sharma, S. Gupta, H. Ahmad, P. Zimniak, A. Radominska, R. Lester, and Y. C. Awasthi. 1991. FEBS [Fed. Eur. Biochem. Soc.] Lett. 281:255257). We now demonstrate that DNP-SG ATPase purified from human lung and erythrocyte membranes catalyzed the hydrolysis of ATP in the presence of doxorubicin and its metabolites. Doxorubicin-stimulated ATP hydrolysis by DNP-SG ATPase was saturable with respect to doxorubicin $\left(K_{\mathrm{m}} 1.2\right.$ and $2.8 \mu \mathrm{M}$ for the lung and erythrocyte enzymes, respectively). Antibodies against DNP-SG ATPase immunoprecipitated the ATP hydrolyzing activity stimulated by doxorubicin, its metabolites, and glutathione conjugates. Inside out vesicles prepared from erythrocyte membranes took up doxorubicin, daunomycin, and vinblastine in an ATP-dependent manner. The uptake was linear with respect to time and vesicle protein, was dependent on ATP and magnesium, was inhibited by heavy metal salts or by heating the vesicles, and was sensitive to both osmolarity and orientation of the vesicles. The transport had an activation energy of $13 \mathrm{kcal} / \mathrm{mol}$, was saturable with respect to both doxorubicin and ATP ( $K_{\mathrm{m}}$ values of $1.8 \mu \mathrm{M}$ and $1.9 \mathrm{mM}$, respectively), and was competitively inhibited by glutathione conjugates as well as by a number of amphiphiles such as daunomycin or vinblastine. Transport was diminished upon coating the vesicles with antibodies against DNP-SG ATPase. Incorporation of increasing amounts of purified DNP-SG ATPase into the vesicles resulted in a linear increase in transport of doxorubicin. These studies demonstrated for the first time that a membrane protein that catalyzed the transport of anionic amphiphilic molecules such as glutathione conjugates could also mediate the transport of weakly cationic antitumor antibiotic, doxorubicin. Notably, the $K_{m}$ of transport was in the range of doxorubi-
\end{abstract}

Address correspondence to Yogesh C. Awasthi, Ph.D., Department of Human Biological Chemistry and Genetics, 2.138 Medical Research Building, University of Texas Medical Branch, Galveston, TX 775551067.

Received for publication 21 July 1993 and in revised form 15 September 1993.

J. Clin. Invest.

(C) The American Society for Clinical Investigation, Inc.

0021-9738/94/03/0958/08 \$2.00

Volume 93, March 1994, 958-965 cin concentration achievable in human serum after intravenous dosing of doxorubicin. (J. Clin. Invest. 1994. 93:958-965.) Key words: doxorubicin • biological transport • glutathione • drug resistance • ethacrynic acid

\section{Introduction}

Doxorubicin hydrochloride $\left(\mathrm{DOX}^{1}\right.$, Adriamycin ${ }^{\circledR}$; Farmitalia, Milan, Italy) is an effective cytoreductive agent for chemotherapy of a variety of neoplasms, but its efficacy is limited by inherent or acquired resistance to its cytocidal effects in cancer cells (1). Studies of DOX resistance have shown that cultured malignant cells resistant to DOX frequently exhibit a multidrug resistant (MDR) phenotype associated with the ATP-dependent efflux of a number of amphiphilic cytotoxins such as DOX, vincristine, and vinblastine (2-7). A number of these malignant cell lines with the MDR phenotype overexpress the P-glycoprotein, a $170-\mathrm{kD}$ glycoprotein product of the $m d r-1$ gene $(5,8)$. The P-glycoprotein has been demonstrated to mediate the ATP-dependent efflux of DOX and other amphiphilic drugs (3-8). Several studies have also documented the existence of an MDR phenotype in malignant cell lines which do not overexpress the P-glycoprotein (9-13). Increased efflux of DOX from MDR cells which do not overexpress P-glycoprotein has also been demonstrated $(5,11-13)$, suggesting that efflux mechanism(s) distinct from P-glycoprotein may be involved in the exclusion of DOX or similar amphiphilic compounds.

ATP-dependent transport of oxidized glutathione and conjugates of GSH and xenobiotics across human erythrocyte membrane is known (14-16), and ATPases catalyzing hydrolysis of ATP in the presence of conjugates of xenobiotics and GSH (17) and oxidized glutathione (18) have been identified in human erythrocyte membrane. A transporter of glutathione-xenobiotic (GS-X) conjugates $(16,17)$ present in the membrane of human erythrocytes and other cells (19) catalyzed ATP hydrolysis in presence of not only GS-X conjugates but also in the presence of several structurally unrelated amphiphilic compounds including estradiol glucuronide, bilirubin ditaurate, lithocholic acid 3-O-glucuronide, and leukotriene $\mathrm{C}_{4}$ methyl ester $(20,21)$. We hypothesize that this GS-X conju-

1. Abbreviations used in this paper: DNP-SG, dinitrophenyl-S-glutathione; DOX, doxorubicin, EA-SG conjugate, ethacrynic acid-glutathione conjugate; G3PD, glyceraldehyde-3-phosphate dehydrogenase; GST, glutathione $S$-transferase; GS-X conjugate, glutathione-xenobiotic conjugate; IOV, inside out vesicle; MDR, multidrug resistant; ROV, right side out vesicle. 
gate transporter, previously designated as DNP-SG ATPase because of its routinely used model substrate dinitrophenyl- $S$ glutathione (DNP-SG) $(16,17)$, may be involved in the energy-dependent efflux of other structurally unrelated amphiphilic compounds such as DOX and may thus be a contributory factor in the mechanism(s) for DOX resistance in malignant cells not overexpressing the P-glycoprotein. This report demonstrates that DNP-SG ATPase purified from human erythrocytes and lung tissue stimulated ATP hydrolysis in the presence of DOX and its metabolites. Furthermore, we have demonstrated that DOX, daunomycin, and vinblastine were taken up by inside out vesicles (IOVs) prepared from membranes of human erythrocytes (which do not express the P-glycoprotein) through an ATP-dependent process. The uptake of DOX was competitively inhibited by substrates of the GS-X pump (DNP-SG ATPase) as well as by prototypical substrates of the P-glycoprotein transporter. The transport of DOX was also inhibited by polyclonal antibodies specific to DNP-SG ATPase. Incorporation of purified DNP-SG ATPase into erythrocyte IOVs more than doubled the rate of DOX transport. These studies suggest a close similarity between the transport mechanisms of DOX and GS-X conjugates. Possible implications of these findings in the mechanisms of drug resistance and strategies of cancer chemotherapy are discussed in this report.

\section{Methods}

Materials. Use of human tissues was approved by the Institutional Review Board. Blood collected from normal subjects and stored for $<1$ wk was obtained from the Blood Bank, and human lung samples from adult subjects without known pulmonary disorders deceased $<8 \mathrm{~h}$ were collected from the autopsy service. Sources of reagents used in the present studies were the same as described previously (21). DOX (Adriamycin ${ }^{\circledast}$ ), doxorubicinone, dihydrodoxorubicinone, deoxydoxorubicinone, dihydrodoxorubicin, and vinblastine sulfate were generously provided by Dr. Sergio Penco (Adria Labs, Milan, Italy). Mitoxantrone and daunorubicin were generously provided by Lederle Laboratories (Pearl River, NY) and by Wyeth Laboratories (Philadelphia, PA), respectively. $\left[14-{ }^{14} \mathrm{C}\right]$ Doxorubicin hydrochloride (sp act 57 $\mathrm{mCi} / \mathrm{mmol}$ ) and $\left[\mathrm{G}^{3} \mathrm{H}\right]$ vinblastine sulfate ( $\mathrm{sp}$ act $11 \mathrm{Ci} / \mathrm{mmol}$ ) were purchased from Amersham Corp. (Arlington Heights, IL). $\left[\gamma^{-32} \mathrm{P}\right]-$ ATP and $\left[\mathrm{G}-{ }^{3} \mathrm{H}\right]$ daunomycin (sp act $1.7 \mathrm{Ci} / \mathrm{mmol}$ ) were procured from Du Pont/New England Nuclear (Boston, MA). ( \pm )-Verapamil hydrochloride was purchased from Sigma Chemical Co. (St. Louis, MO). Taurodeoxycholate and taurolithocholate (sodium salts) were purchased from Calbiochem-Novabiochem Corp. (La Jolla, CA). The conjugate of 1-chloro-2,4-dinitrobenzene and GSH, 2,4-dinitrophenyl$S$-glutathione (DNP-SG), was synthesized enzymatically, purified, and authenticated according to our previously published method (22) and was linked to $\mathrm{CNBr}$-activated Sepharose $4 \mathrm{~B}$ according to a method described previously (21). GSH conjugate of ethacrynic acid (EA-SG) was synthesized and authenticated as described by us previously (23). Polyclonal antibodies against human erythrocyte DNP-SG ATPase and glyceraldehyde 3-phosphate dehydrogenase, raised in rabbits, were the same as those used in previous studies (19). The IgG fractions were isolated from these antibodies and the preimmune serum by DEAEcellulose ion-exchange chromatography and were purified further using a column of protein A bound to $\mathrm{CNBr}$-activated Sepharose $6 \mathrm{MB}$.

Enzyme assays and purification of DNP-SG ATPase. ATPase activity in the presence of DNP-SG, DOX, and other substrates was determined according to the method used by us previously (21). $1 \mathrm{mU}$ of enzyme activity was defined as $1 \mathrm{nmol}$ ATP hydrolysis $/ \mathrm{min}$ at $37^{\circ} \mathrm{C}$. Purifications of DNP-SG ATPase from human lung were performed at $4^{\circ} \mathrm{C}$ according to a previously described protocol (21). The details of purification of DNP-SG ATPase from human erythrocytes have also been reported in our previous studies (19). Because of interference by lubrol PX (Sigma Chemical Co. ) used during purification, protein was estimated by the method of Minamide and Bamburg (24). SDSPAGE, Western blot analysis, and immunotitration of DNP-SG ATPase were performed by methods used by us previously $(19,21)$.

Preparation of IOVs and right side out vesicles (ROVs) from human erythrocyte membranes. Preparation of human erythrocyte vesicles was carried out by the method of Steck and Kant (25) in which human erythrocyte ghosts prepared according to the method of Dodge et al. (26) were diluted in $40 \mathrm{vol}$ of $0.5 \mathrm{mM}$ sodium phosphate buffer, $\mathrm{pH} 8.0$, and were allowed to incubate overnight at $4^{\circ} \mathrm{C}$. The pellet obtained by centrifugation at $28,000 \mathrm{~g}$ for $1 \mathrm{~h}$ was resuspended in the same buffer, and vesiculation was performed by repeatedly passing the suspension through a 27 -gauge needle. Vesiculation performed in presence or absence of $250 \mathrm{mM}$ sucrose resulted in vesicles with similar yield and transport properties. The IOVs were purified either by density gradient centrifugation at $105,000 \mathrm{~g}$ for $2 \mathrm{~h}$ over a dextran barrier $(1.03 \mathrm{~g} / \mathrm{ml})$ during which the ROVs and unsealed ghosts pelleted to the bottom, or by passing the mixture of vesicles over a column $(1 \mathrm{~cm} \times 5$ $\mathrm{cm}$ ) of wheat germ agglutinin bound to $\mathrm{CNBr}$-activated Sepharose 4B. In the latter procedure, the ROVs were almost quantitatively retained by the column, whereas the IOVs were recovered in the flow-through fraction of the column. The ROVs were eluted from the column with $50 \mathrm{mM}$ methyl- $\alpha$-D-mannopyranoside. The purities of the IOVs and ROVs were estimated by comparing acetylcholinesterase activities in the erythrocyte ghosts and the vesicles using the assay described by Ellman et al. (27). Both IOVs and ROVs were finally suspended in buffer containing $250 \mathrm{mM}$ sucrose in $10 \mathrm{mM}$ Tris- $\mathrm{HCl}, \mathrm{pH} 7.4$.

Transport studies with DOX and other P-glycoprotein substrates. For the transport studies, stock solutions of $10 \mathrm{mM} \mathrm{MgCl}_{2}$ and $20 \mathrm{mM}$ ATP were prepared in buffer containing $250 \mathrm{mM}$ sucrose and $10 \mathrm{mM}$ Tris- $\mathrm{HCl}, \mathrm{pH} 7.4$. The $\mathrm{pH}$ of the ATP solution was adjusted to 7.4 with $0.1 \mathrm{~N} \mathrm{NaOH}$. The osmolarity of all solutions was measured using a microosmometer (model 3MO; Baxter Scientific, McGraw Park, IL). The reaction mixture consisted of IOV protein $(0.5-14 \mu \mathrm{g}), 10 \mathrm{mM}$ Tris- $\mathrm{HCl}, \mathrm{pH} 7.4,250 \mathrm{mM}$ sucrose, $4 \mathrm{mM} \mathrm{MgCl}$, and either $2 \mathrm{mM}$ ATP or an equiosmolar concentration of $\mathrm{NaCl}(3 \mathrm{mM})$. Since ROVs prepared by wheat germ agglutinin affinity chromatography contained methyl- $\alpha$-D-mannopyranoside, the experiments in which the transport activities of the IOVs and ROVs were compared included equal concentrations of methyl- $\alpha$-D-mannopyranoside in incubation mixtures containing IOVs. To start the reaction, $20 \mu \mathrm{l}$ of radiolabeled $\left[14-{ }^{14} \mathrm{C}\right]-$ DOX $(75,000 \mathrm{cpm} / \mathrm{nmol})$ dissolved in Tris/sucrose buffer was added to achieve a final volume of reaction mixture of $100 \mu \mathrm{l}$. The reaction mixtures were incubated at $37^{\circ}$ for $10 \mathrm{~min}$, and free DOX was separated from the vesicles. Because DOX is a freely membrane-permeable amphiphilic compound, a method for the rapid separation of IOVs from free DOX to minimize the leakage of DOX from IOVs was necessary. We initially used Penefsky's method (28) in which the separation of the IOVs from free DOX was achieved by Sephadex G-50 size exclusion chromatography in a $1-\mathrm{ml}$ syringe by centrifugation. The results obtained by this method, however, were not readily reproducible between experiments and varied significantly probably because of small differences in filtration times and possibly because of differences in the packing of the Sephadex beads. We conjectured that these differences could arise due to the leakage of DOX from vesicles into the surrounding medium because as the IOVs are separated from free DOX, the concentration gradient of DOX between the IOVs and surrounding buffer increased, causing an increased leakage of DOX from the IOVs. To minimize the diffusion of DOX from the IOVs, we devised a rapid membrane filtration method employing a 96-well plate multiscreen assay system (Millipore Corp., Bedford, MA) in which $0.45-\mu \mathrm{m}$ pore size nitrocellulose membranes are individually attached to the bottom of each of the wells. Rapid filtration of the incubation mixtures used in transport studies to separate free DOX from the IOVs was achieved by attaching the plate to a vacuum device. During standardization of this method, we were able to get complete separation of free DOX and 
Table I. Purification of DNP-SG ATPase from Human Lung

\begin{tabular}{|c|c|c|c|c|c|c|c|}
\hline \multirow[t]{2}{*}{ Fraction } & \multirow{2}{*}{$\begin{array}{c}\text { Volume } \\
\mathrm{ml}\end{array}$} & \multicolumn{2}{|c|}{ Activity* } & \multirow{2}{*}{$\begin{array}{l}\text { Protein } \\
m g / m l\end{array}$} & \multirow{2}{*}{$\frac{\text { Specific activity }}{\begin{array}{l}\mathrm{mU} / \mathrm{mg} \\
\text { protein }\end{array}}$} & \multirow{2}{*}{$\begin{array}{c}\text { Yield } \\
\%\end{array}$} & \multirow{2}{*}{$\begin{array}{c}\text { Purification } \\
\text { Fold }\end{array}$} \\
\hline & & $m U / m l$ & Total $(m U)$ & & & & \\
\hline Membrane & 10 & 17.4 & 174.0 & 10.1 & 1.7 & 100 & - \\
\hline Lubrol extract & 9 & 17.2 & 154.8 & 10.2 & 1.7 & 89 & - \\
\hline DNP-SG elute & 15 & 1.5 & 22.5 & 0.084 & 18.0 & 13 & 11 \\
\hline $\begin{array}{l}\text { G3PD immunoaffinity } \\
\text { unadsorbed fraction }\end{array}$ & 16 & 1.3 & 20.8 & 0.062 & 21.0 & 12 & 12 \\
\hline
\end{tabular}

$40 \mathrm{~g}$ human lung as starting material was used for the purification.

* $1 \mathrm{mU}$ of enzyme catalyzed $1 \mathrm{nmol}$ ATP hydrolyzed/min at $37^{\circ} \mathrm{C}$.

IOVs within $15 \mathrm{~s}$ from the incubation mixtures containing up to 140 $\mu \mathrm{g} / \mathrm{ml}$ of vesicle protein. Nonspecific binding of DOX to cellulose nitrate membrane was minimized by washing the membrane with 3.4 $\mathrm{mM}$ DOX before experiments and was unaffected by the presence or absence of ATP. After filtration, the bottoms of the membranes were blotted dry with filter paper, were punched out and placed in liquid scintillation counting vials containing $10 \mathrm{ml}$ of scintillation fluid (Hydrofluor/Betafluor, 1:1 [ vol/vol]; National Diagnostics, Atlanta, GA, and Manville, NJ, respectively), were vortexed thoroughly, were allowed to stand for $1 \mathrm{~h}$ at room temperature, and were counted in a liquid scintillation counter (LS-6800; Beckman Instruments, Inc., Fullerton, CA). ATP-dependent uptake of DOX was determined by subtracting the radioactivity (cpm) of the control without ATP from that of the experimental containing ATP, and the transport of DOX was calculated in terms of picomoles per minute per milligram IOV protein. The transport of daunomycin and vinblastine were performed in a similar manner using [G- ${ }^{3} \mathrm{H}$ ] daunomycin ( $\mathrm{sp}$ act $52,000 \mathrm{cpm} / \mathrm{nmol}$ ) and $\left[\mathrm{G}-{ }^{3} \mathrm{H}\right.$ ] vinblastine sulfate ( $\mathrm{sp}$ act $46,000 \mathrm{cpm} / \mathrm{nmol}$ ).

Kinetic studies and inhibition of DOX transport. The kinetics of transport of DOX, daunomycin, and vinblastine were measured in separate experiments at various concentrations of ATP or these substrates keeping the other fixed, and double reciprocal plots of $1 / v$ vs $1 /$ [S] were generated to determine $K_{\mathrm{m}}$ values. The inhibitory effect of the GSH conjugates (DNP-SG and EA-SG), and of bilirubin ditaurate, taurolithocholate, taurodeoxycholate, mitoxantrone, daunorubicin, verapamil, vinblastine, and cyclosporin $A$ on the transport of DOX was investigated by comparing its transport in the absence and presence of different concentrations of these compounds. The reaction mixtures were preincubated with the inhibitor for $5 \mathrm{~min}$ at $37^{\circ} \mathrm{C}$ before addition of the substrates. The $I_{50}$ values (the concentration of the inhibitor giving 50\% inhibition of the rate of DOX transport) were determined from the plots of percentage of control DOX transport vs inhibitor concentration. The nature of inhibition was determined by double reciprocal plots; the $K_{\mathrm{i}}$ was determined by the replots of the double reciprocal plots.

Reconstitution experiments. Erythrocyte IOVs were sonicated in a sonifier cell disruptor at $40 \mathrm{~W}$ for $1 \mathrm{~min}$ on ice in the presence of increasing amounts of purified DNP-SG ATPase protein. Equal amounts of albumin were added to control vesicles and treated similarly. After a 2 -h incubation at $4^{\circ} \mathrm{C}$, these vesicle preparations were subjected to wheat germ agglutinin chromatography as described above. DOX transport studies were performed as described above on the IOVs obtained in the unadsorbed fraction of wheat germ agglutinin chromatography as described in this section.

\section{Results}

The results of purification of DNP-SG ATPase from human lung, presented in Table I, indicate that the enzyme could be purified with an overall yield of $13 \%$, similar to that previously observed during purification of this enzyme from muscle (21). A major portion of activity was lost at the affinity chromatography step, probably because of enzyme inactivation. Traces of glyceraldehyde-3-phosphate dehydrogenase (G3PD) which have previously been found to be associated with DNP-SG ATPase purified from human erythrocytes (19) were removed by immunoaffinity chromatography using anti-G3PD antibodies linked to CNBr-activated Sepharose 4B. The purified enzyme recovered in the flow-through fraction of the immunoaffinity column showed a single band on SDS-PAGE (Fig. $1 A$ ) with a subunit $M_{\mathrm{r}}$ value of $\sim 38 \mathrm{kD}$, which was similar to the $M_{\mathrm{r}}$ value of human erythrocyte and human muscle DNP-SG ATPase reported by us previously $(19,21)$. In Western blots (Fig. $1 B$ ), the enzyme was recognized by polyclonal antibodies raised in rabbits against human erythrocyte DNP-SG ATPase. The specific activity of the purified enzyme from lung towards DNP-SG was comparable with that reported for the erythrocyte and muscle enzymes $(19,21)$. In parallel experiments, human erythrocyte DNP-SG ATPase was also purified. ATP hydrolysis catalyzed by the lung and erythrocyte enzymes in the presence of various $S$-alkyl- and $S$-aryl-glutathione conjugates ( Table II) was similar to that previously reported for human muscle DNP-SG ATPase (21). In general, the long alkyl chain conjugates exhibited higher activity than the conjugates
A

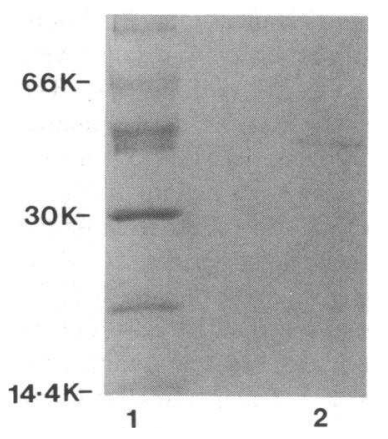

B

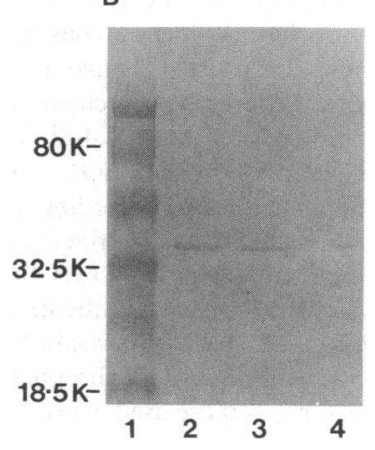

Figure 1. Homogeneity and immunoreactivity of purified human lung DNP-SG ATPase. SDS-PAGE of the purified human lung DNP-SG ATPase $(A)$ with standard protein markers and purified human lung DNP-SG ATPase present in lanes 1 and 2, respectively. Immunoreactivity of human lung DNP-SG ATPase in Western blot analysis with prestained standard marker proteins, DNP-SG affinity eluate, unadsorbed fraction of anti-G3PD immunoaffinity column, and crude membrane lubrol extract present in lanes $1-4$, respectively. 
Table II. Activity of DNP-SG ATPase towards Various Substrates

\begin{tabular}{|c|c|c|c|c|c|c|}
\hline \multirow[b]{2}{*}{ Activators } & \multicolumn{2}{|c|}{ Specific activity* } & \multicolumn{2}{|c|}{$K_{\mathrm{m}}$} & \multicolumn{2}{|c|}{$V_{\max }$} \\
\hline & Lung & Erythrocytes & Lung & Erythrocytes & Lung & Erythrocytes \\
\hline & \multicolumn{2}{|c|}{$m U / m g$ protein } & \multicolumn{2}{|c|}{$\mu M$} & \multicolumn{2}{|c|}{$\begin{array}{c}n m o l / m i n \\
\text { per milligram protein }\end{array}$} \\
\hline DNP-SG & 20.3 & 23.1 & 125.2 & 80.1 & 33 & 32 \\
\hline Doxorubicin & 25.9 & 48.4 & 1.2 & 2.8 & 25 & 48 \\
\hline Doxorubicinone & 24.8 & 29.4 & 5.9 & 5.8 & 40 & 48 \\
\hline Dihydrodoxorubicinone & 27.2 & 38.7 & 3.4 & 5.2 & 30 & 51 \\
\hline Deoxydoxorubicinone & 22.5 & 32.5 & 2.9 & 7.6 & 33 & 63 \\
\hline Dihydrodoxorubicin & 18.6 & 39.0 & 3.5 & 2.0 & 28 & 53 \\
\hline$S$-methyl glutathione & 8.3 & 9.4 & ND & ND & ND & ND \\
\hline$S$-(n-propyl) glutathione & 9.0 & 9.8 & ND & ND & ND & ND \\
\hline$S$-( $n$-pentyl) glutathione & 12.7 & 14.2 & ND & ND & ND & ND \\
\hline$S$-(n-decyl) glutathione & 13.9 & 15.0 & ND & ND & ND & ND \\
\hline$S$-(p-chlorophenacyl) glutathione & 15.9 & 18.8 & ND & ND & ND & ND \\
\hline$S$-( $p$-nitrobenzyl) glutathione & 18.1 & 23.9 & ND & ND & ND & ND \\
\hline
\end{tabular}

${ }^{*} 1 \mathrm{mU}$ of enzyme catalyzed, $1 \mathrm{nmol}$ ATP hydrolyzed/min at $37^{\circ} \mathrm{C}$. ND, not determined. Values represent mean of $n=3$ determinations; the relative standard deviation was $<4 \%$ in all cases.

with shorter alkyl chain. These results indicated that the lung enzyme was similar and probably identical to the DNP-SG ATPase characterized previously from human erythrocytes and muscle $(19,21)$.

Catalysis of ATP hydrolysis in the presence of DOX. Purified human lung DNP-SG ATPase not only catalyzed the hydrolysis of ATP in the presence of DOX and its metabolites, but comparatively higher ATPase activity of the enzyme was observed with these compounds as compared to that with GSH conjugates (Table II). DOX-stimulated ATP hydrolysis by DNP-SG ATPase purified from both erythrocytes and lung was linear with the amount of the purified enzyme used and with the incubation time for at least $60 \mathrm{~min}$. The results of these experiments with the erythrocyte enzyme are presented in Fig. 2, $A$ and $B$; similar results were obtained with the lung enzyme. DOX-stimulated ATPase activity of the enzyme was abolished when the enzyme was preheated at $90^{\circ} \mathrm{C}$ for $5 \mathrm{~min}$, a result consistent with the previously reported heat inactivation of this enzyme (20).

The DOX- and doxorubicinone-stimulated ATP hydrolyzing activity of the purified erythrocyte enzyme was found to be immunoprecipitated ( 77 and $91 \%$, respectively) by anti-DNPSG ATPase antibodies in immunotitration experiments in which preimmune serum did not significantly affect the activity (Fig. $2 C$ ). In parallel experiments, the ATPase activity stimulated by the GSH conjugate (DNP-SG) used as a positive control was also immunoprecipitated to the extent of $82 \%$ (Fig. $2 C$ ). Similar results were obtained with the purified enzyme from human lung (data not presented).

ATP hydrolysis by the human lung and erythrocyte DNP. SG ATPase by DOX and its metabolites was saturable at higher concentrations of these compounds. Linearity of double reciprocal plots was observed for both human lung and erythrocyte enzymes with DOX and its metabolites as substrates (data not presented). The $K_{\mathrm{m}}$ and $V_{\max }$ values of the erythrocyte and lung enzymes for each compound are shown in Table II. The $K_{\mathrm{m}}$ values of the erythrocyte and lung enzymes for these compounds were much lower than those for GS-X conjugates and ranged from 1.2 to $7.6 \mu \mathrm{M}$. It is important to note that the $K_{\mathrm{m}}$ values of the enzymes for DOX and its metabolites were within the range of peak serum DOX concentrations achievable with conventional doses of DOX during chemotherapy.

Transport of DOX. IOVs prepared from erythrocyte membranes by the method described by Steck and Kant (25) were $\sim 80 \%$ pure as determined by acetylcholinesterase latency (27). Results of transport studies with the IOVs made in the absence or presence of $250 \mathrm{mM}$ sucrose were similar, with the exception that the DOX transport activity was more stable at $4^{\circ} \mathrm{C}$ in the IOVs vesiculated in the presence of sucrose. Before performing transport studies, osmolarity was measured on all

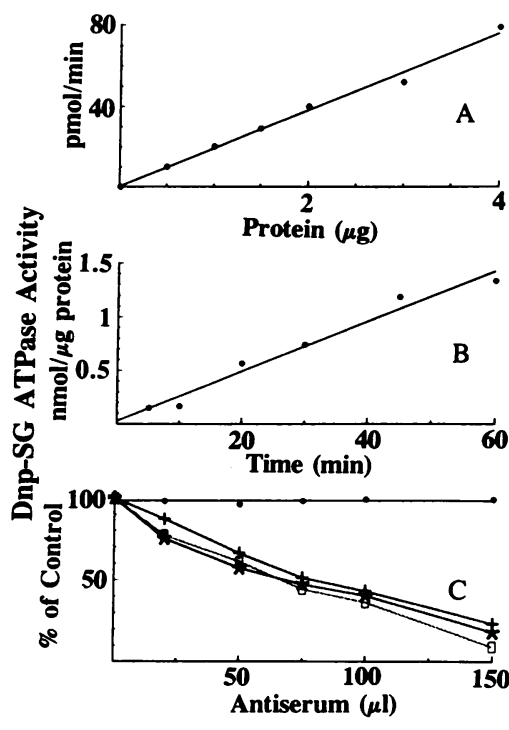

Figure 2. Human erythrocyte DNP-SG ATPase activity. DOX-stimulated ATPase activity of the purified transporter with respect to varying concentrations of protein and with respect to time of incubation of the reaction mixture are presented in $A$ and $B$ respectively. DOX concentration used in these studies was $3.6 \mu \mathrm{M}$. The immunoprecipitation of DNP-SG $(\star)$, doxorubicin $(+)$, and doxorubicinone ( $\square$ ) stimulated ATPase activity of the purified enzyme with anti-DNP-SG ATPase antibodies and the effect

of preimmune serum (•) on DOX-stimulated ATPase activity are presented in $C$. The concentrations of DNP-SG, doxorubicin, and doxorubicinone used in these studies were 120,4 , and $20 \mu \mathrm{M}$, respectively. 


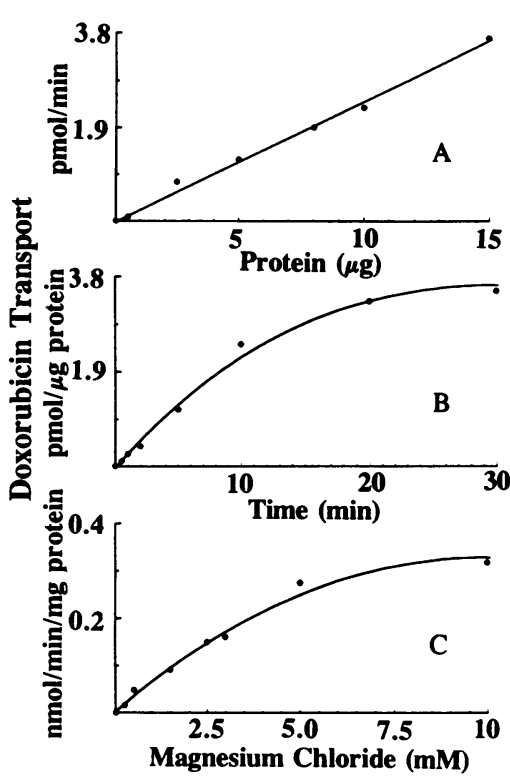

magnesium dependence, transport was measured after a 10-min incubation $(A$ and $C$ ). Protein concentration was $14.5 \mu \mathrm{g} / 100 \mu \mathrm{l}$ reaction mixture for studies of time and magnesium dependence ( $B$ and $C$ ).

solutions to ensure that the osmolarity of the blank and reaction mixtures used for transport studies were identical. Using either $\mathrm{NaCl}$ or ADP in the controls to compensate for the osmolarity of ATP in the experimental reaction mixtures yielded similar results. Increased ATP-dependent uptake of DOX by the IOVs, as indicated by the association of $\left[{ }^{14} \mathrm{C}\right] \mathrm{DOX}$ radioactivity with the IOVs, was consistently seen in the presence of ATP as compared with the IOVs incubated in the presence of either ADP or $\mathrm{NaCl}$. ATP-dependent uptake of DOX by the IOVs was linear with respect to the IOV protein concentration (Fig. $3 A$ ). These results were observed regardless of whether the Sephadex G-50 column filtration method of Penefsky (28) or the membrane filtration method developed by us was used to separate IOVs from free DOX in the reaction mixtures. However, the precision was significantly better with the nitrocellulose membrane filtration method, and thus, the data presented in this report are those obtained using the latter method. The ATP-dependent DOX uptake by the IOVs was rapid and approximately linear for the first $15 \mathrm{~min}$, with an initial rate of $\sim 300 \mathrm{pmol} / \mathrm{min}$ per milligram of protein (Fig. $3 \mathrm{~B}$ ). Because outward diffusion of DOX (a freely membrane-permeable compound ) should start as soon as a concentration gradient of DOX across the IOV membrane was generated, the stoichiometry between DOX uptake and ATP consumption was difficult to determine. The results presented in Fig. $3 C$ showed that the ATP-dependent transport of DOX was magnesium dependent. ATP-dependent DOX transport by the IOVs was inhibited $\sim 60 \%$ by $100 \mu \mathrm{M} \mathrm{HgCl}_{2}$ and $47 \%$ by $100 \mu \mathrm{M} \mathrm{CrCl}_{3}$.

Because DOX is known to have a high degree of affinity for membranes (29), it is important to rule out that the observed accumulation of DOX in the IOVs observed during the present studies did not result because of a nonspecific binding of DOX to the IOV membrane or to the nitrocellulose filter, even though the ATP dependence of the process argues against these possibilities. Transport experiments were therefore performed to compare the uptake of DOX by ROVs and IOVs. We reasoned that if DOX was transported from inside the erythrocytes by ATP-dependent processes then ROVs should not show the ATP-dependent uptake of DOX. If, on the other hand, DOX incorporation in IOVs observed in our experiments was because of binding of DOX to the membrane, both IOVs and ROVs should accumulate DOX. Taking advantage of the presence of carbohydrate residues exclusively on the noncytoplasmic surface of membranes, the ROVs and IOVs were separated from the mixture of vesicles by wheat germ agglutinin column chromatography (Fig. 4). Measurement of acetylcholinesterase activity in the IOVs recovered in the flowthrough fractions of the column and the ROVs obtained by eluting the column with methyl- $\alpha$-D-mannopyranoside $(1.3$ and $5.6 \mu \mathrm{mol} / \mathrm{min}$ per milligram protein for IOVs and ROVs, respectively) indicated enrichment of ROVs in the methyl- $\alpha$ D-mannopyranoside eluate. Treatment of IOVs and ROVs with Triton X-100 resulted in similar acetylcholinesterase activities in both fractions. IOVs purified by the wheat germ agglutinin column chromatography showed the ATP-dependent uptake of DOX at a rate of $280 \mathrm{pmol} / \mathrm{min}$ per milligram protein which was similar to that observed with the IOVs prepared by Steck and Kant's method (25). The ROVs, on the other hand, showed an uptake rate of $30 \mathrm{pmol} / \mathrm{min}$ per milligram ROV protein. When equal amounts of ROVs and IOVs were mixed and the mixtures were used for transport studies, the ATP-dependent uptake of DOX by the mixed vesicles was found to be $128 \mathrm{pmol} / \mathrm{min}$ per milligram protein, which was approximately half of that seen with IOVs alone. These results, demonstrating a minimal accumulation of DOX in the ROVs as compared with that in the IOVs, ruled out the possibility that observed ATP-dependent accumulation of DOX in the IOVs was because of increased nonspecific binding in the presence of ATP. The intravesicular accumulation of DOX was further confirmed by studying the relationship between the intravesicular space of the IOVs and DOX accumulation. Experiments were carried out to determine the ATP-dependent uptake of DOX by the IOVs in the presence of increasing concentrations of sucrose, which would result in gradual shrinkage of the IOVs and decreased intravesicular space. The results of these experiments presented in Fig. 5 indicated that ATP-dependent uptake of DOX by the IOVs decreased with the increasing concentration of sucrose in the incubation medium. Extrapolation to infinite sucrose concentration, i.e., zero intravesicular volume, indicated that $\sim 35 \%$ of the observed uptake was because of nonspecific binding, a reasonable number for a relatively hydrophobic compound such as DOX.

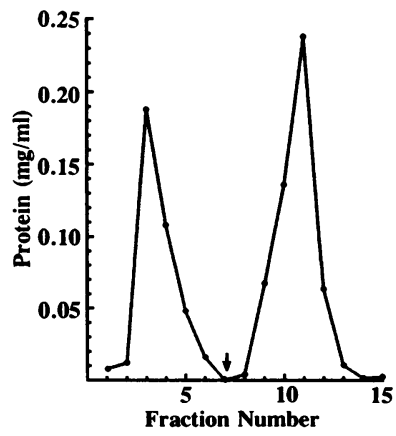

Figure 4. Separation of IOVs and ROVs by wheat germ agglutinin affinity chromatography. Fractions of $0.5 \mathrm{ml}$ each were collected after application of vesicle protein to a wheat germ agglutinin column with a constant flow rate of $6 \mathrm{ml} /$ h. The arrow indicates the time at which elution with methyl $\alpha$-Dmannopyranoside was begun. 


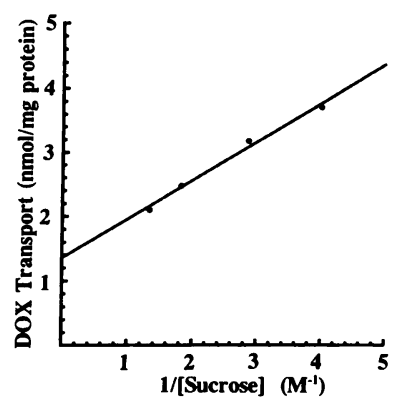

Figure 5. Osmotic sensitivity of DOX transport in erythrocyte IOVs. Total ATP-dependent uptake of DOX after 30 min was measured in the presence of 250 $950 \mathrm{mM}$ sucrose, and the uptake was plotted vs the inverse of sucrose concentration. Linear regression was used to extrapolate the DOX binding to the vesicles at infinite sucrose concentration.

The dependence of DOX uptake on ATP and on proper membrane sidedness indicated that the transport was mediated by an ATP-dependent pump. This was further verified by determining the activation energy of the transport, which is compatible with an enzymatic process, and by establishing the heat sensitivity of the process. The ATP-dependent DOX transport by IOVs was shown to be temperature dependent with rates of 21,127 , and $217 \mathrm{pmol} / \mathrm{min}$ per milligram protein at 4,20 , and $37^{\circ} \mathrm{C}$, respectively, yielding an activation energy of $\sim 13 \mathrm{kcal} /$ mol. Inactivation of the transporter upon heating was demonstrated in two separate experiments. IOVs preheated at $45^{\circ} \mathrm{C}$ for 1 and $4 \mathrm{~h}$ transported DOX at 14.0 and $2.5 \mathrm{pmol} / \mathrm{min}$ per milligram protein, corresponding to 6 and $1 \%$ of control transport rate, respectively. In other experiments, IOVs were immersed in boiling water for $5 \mathrm{~min}$, were cooled to room temperature, and were revesiculated by sonication. IOVs were then separated from the mixture of vesicles using a wheat germ agglutinin column which quantitatively retained ROVs. The transport of DOX in the heat-inactivated IOVs thus prepared was completely abolished ( $<1 \%$ of control $)$. The kinetic characterization of the transport mechanism for DOX during the present studies included the determination of the $K_{\mathrm{m}}$ and $V_{\max }$ values for its substrates, DOX and ATP (Fig. 6, $A$ and $B$, respectively). The $K_{\mathrm{m}}$ of the transporter for ATP was found to be 1.85 $\mathrm{mM}$, which was in the same range as that reported for GSH conjugate transporter $(16,17,21)$. The $K_{\mathrm{m}}$ of the transporter for DOX was found to be $1.7 \mu \mathrm{M}$, which was closely similar to the $K_{\mathrm{m}}$ values (Table II) of the ATP-hydrolyzing activity of DNP-SG ATPase for DOX purified from either erythrocytes or

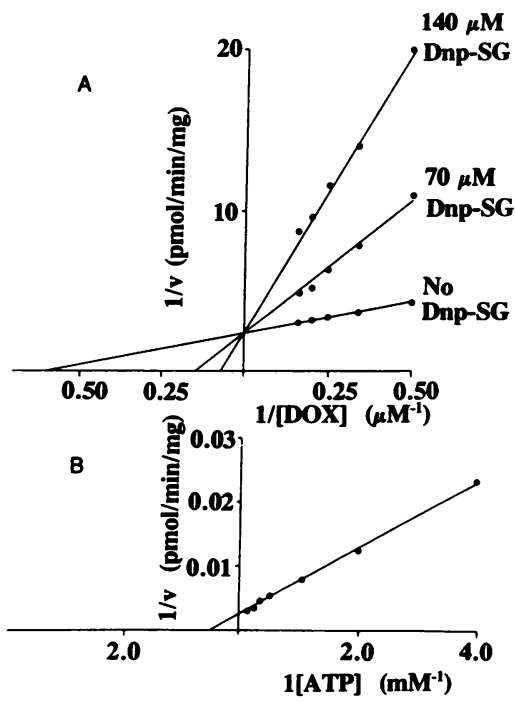

Figure 6. Kinetics of DOX transport in human erythrocyte IOVs. Competitive inhibition of DOX transport by DNP-SG $(A)$ and the $K_{\mathrm{m}}$ of ATP for DOX transport $(B)$ are presented. The concentration of DOX used for determination of the $K_{\mathrm{m}}$ of ATP was $3.6 \mu \mathrm{M}$. lung $(1.7 \mu \mathrm{M})$. Furthermore, these $K_{\mathrm{m}}$ values were within the range of peak DOX serum concentrations after conventional intravenous dosing of DOX in humans (30) during cancer chemotherapy. In a manner similar to that of DOX transport, a saturable ATP-dependent transport by the IOVs was also observed for daunomycin $\left(K_{\mathrm{m}}=3.3 \mu \mathrm{M}\right.$, and $V_{\max }=200 \mathrm{pmol} /$ min per milligram protein $)$ and vinblastine $\left(K_{\mathrm{m}}=2.9 \mu \mathrm{M}\right.$, and $V_{\max }=212 \mathrm{pmol} / \mathrm{min}$ per milligram protein). Since the presence of DNP-SG ATPase has been demonstrated in all the human tissues examined so far, these results strongly suggested that this transport mechanism may be an important and physiologically relevant protective mechanism against the amphiphilic cytotoxins such as DOX, daunomycin, or vinblastine.

Inhibition of DOX transport by substrates of P-glycoprotein, GSH conjugates, and the antibodies against DNP-SG ATPase. The overlap between the transport mechanism(s) for DOX and GSH conjugates was corroborated by the results of experiments showing that the ATP-dependent uptake of DOX by IOVs was competitively inhibited by DNP-SG (Fig. $6 \mathrm{~A}$ ) and EA-SG (data not presented). The inhibitory effects of several classes of amphiphilic compounds including anthracyclines (mitoxantrone and daunorubicin), vinca alkaloid (vinblastine), calcium channel blocker (verapamil), bilirubin conjugate (bilirubin ditaurate), bile acid conjugates (taurolithocholate and taurodeoxycholate), and cyclosporin A on the transport of DOX were also examined. The inhibition constants and nature of inhibition for these compounds are presented in Table III. It is noteworthy that the known substrates of P-glycoprotein (vinblastine, daunomycin, vincristine, and mitoxantrone) and the known substrates of the DNP-SG ATPase (bilirubin ditaurate, DNP-SG, and EA-SG) were found to inhibit DOX transport in a competitive manner. In contrast, the amphiphilic bile salts which do not stimulate ATP hydrolysis by DNP-SG ATPase (20) were not inhibitory towards this transporter at concentrations up to $100 \mu \mathrm{M}$ used in these studies. Interestingly, cyclosporin A, a compound which can reverse MDR in cells with or without P-glycoprotein overexpression, also inhibited this enzyme, albeit in a noncompetitive manner. Additional evidence for the shared transport mechanisms of DOX and GSH conjugates was provided by a significant inhibition of DOX transport in IOVs preincubated for 5 min with
Table III. Inhibition of DOX Transport by Various Amphiphilic Compounds

\begin{tabular}{|c|c|c|c|}
\hline Inhibitors & $I_{50} *$ & $K_{\mathbf{i}}$ & $\begin{array}{l}\text { Type of inhibition } \\
\text { with respect to DOX }\end{array}$ \\
\hline & $\mu M$ & $\mu M$ & \\
\hline Vinblastine & 12.0 & 9.5 & Competitive \\
\hline Verapamil & 10.0 & 8.3 & Competitive \\
\hline Mitoxantrone & 15.0 & 12.0 & Competitive \\
\hline Bilirubin ditaurate & 85.0 & 60.0 & Competitive \\
\hline Daunorubicin & 3.0 & 1.8 & Competitive \\
\hline Cyclosporin A & 90.0 & 75.0 & Noncompetitive \\
\hline Taurolithocholate & Not inhibitory ${ }^{\ddagger}$ & - & - \\
\hline Taurodeoxycholate & Not inhibitory ${ }^{\ddagger}$ & - & - \\
\hline
\end{tabular}

* The $I_{50}$ value is the concentration of inhibitor giving $50 \%$ inhibition of DOX transport. Details are given in the text. ${ }^{\ddagger}$ Concentrations of these compounds up to $100 \mu \mathrm{M}$ were used in inhibition studies. 
polyclonal antibodies against the human erythrocyte DNP-SG ATPase. Whereas $\sim 45 \%$ inhibition of the transport of DOX was observed in the IOVs coated with anti-DNP-SG ATPase antibodies, the preimmune serum showed no effect on the transport of DOX by the IOVs (Fig. 7). Taken together, these results provided strong evidence that the ATP-dependent transport of GSH conjugates and DOX from human erythrocytes, lung, and possibly other tissues was mediated through shared mechanism(s) and that DNP-SG ATPase was a common component of this mechanism(s).

Reconstitution studies. The purified DNP-SG ATPase protein used for reconstitution studies was essentially homogeneous as seen in SDS-PAGE and was the only protein recognized by anti-DNP-SG ATPase antibodies in Western blot analysis. This protein was not recognized by the $\mathrm{C} 219$ antibody (which recognizes P-glycoprotein) in Western blot analysis (data not presented). The results of DOX transport studies in IOVs reconstituted in the presence of increasing amounts of purified DNP-SG ATPase are presented in Fig. 8. These results show a linear increase in DOX transport activity in IOVs reconstituted in the presence of increasing amounts of the purified DNP-SG ATPase. Activity was unaffected in IOVs reconstituted in the presence of increasing amounts of albumin.

\section{Discussion}

Although the DNP-SG ATPase was initially thought to be a transporter of GS-X conjugates (17), structurally unrelated anionic compounds were later added to its list of substrates (20). Present studies indicate a much broader role for the DNP-SG ATPase as a xenobiotic transporter because we now demonstrate that the weakly cationic compounds (doxorubicin, dihydrodoxorubicin, daunomycin, and vinblastine) and uncharged compounds (doxorubicinone, deoxydoxorubicinone, and dihydrodoxorubicinone) also stimulate ATP hydrolysis by DNP-SG ATPase, and that DOX, daunomycin, and vinblastine are transported across the erythrocyte membranes (which do not express $m d r$-1 gene product P-glycoprotein) through an ATP-dependent transport mechanism most likely mediated by DNP-SG ATPase. This exceptionally broad substrate specificity and its wide tissue distribution indicate that this versatile transport mechanism may function normally as an important efflux mechanism for both xenobiotics and endobiotics. Its ability to transport the lipophilic xenobiotics and the amphiphilic products of phase II detoxification puts it in a unique position. This is probably the only cellular defense sys-

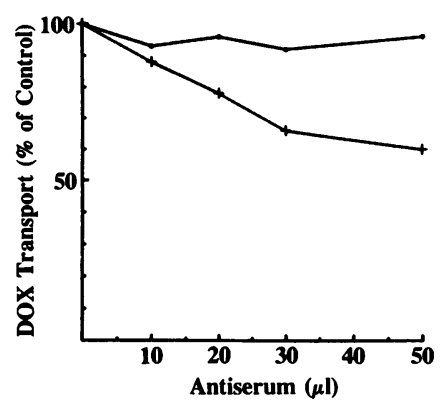

Figure 7. The effect of antiDNP-SG ATPase antibodies on the rate of DOX transport in erythrocyte IOVs. DOX transport rate for the first 10 $\min$ at $3.6 \mu \mathrm{M}$ DOX and 14.5 $\mu \mathrm{g} \mathrm{IOV}$ protein in the presence of equal amounts of either preimmune serum $(\bullet)$ or antiDNP-SG antibody $(+)$ protein are shown as the percentage of control containing neither the preimmune serum nor the anti-DNP-SG ATPase antibody.

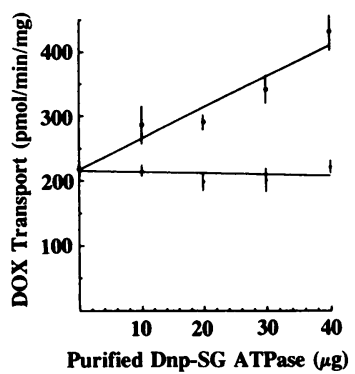

Figure 8. Transport of DOX in erythrocyte IOVs reconstituted with purified erythrocyte DNP-SG ATPase. DOX transport rate of erythrocyte IOVs stored for $<1$ wk at $4^{\circ} \mathrm{C}$ and sonicated in the presence of varying amounts of the purified DNP-SG ATPase protein $(O)$ or equal quantities of albumin $(\bullet)$. DOX and IOV protein concentrations used in these studies were $3.6 \mu \mathrm{M}$ and $14 \mu \mathrm{g} / 100$ $\mu \mathrm{l}$ reaction mixture, respectively.

tem against toxic compounds characterized so far which may be involved in the protection against the toxic effects of amphiphilic toxins as well as their phase I and II biotransformation products.

The wide tissue distribution (19) and ability to transport DOX with a $K_{\mathrm{m}}$ in the range of clinically achievable serum DOX concentrations is particularly interesting, suggesting that this transport mechanism may be important in protecting normal or malignant tissues from the toxic effects of DOX. The cytotoxicity of DOX is closely related to the degree of its intracellular accumulation (31). Increased expression of P-glycoprotein, the best characterized mechanism for decreasing intracellular accumulation of DOX (3-8), has been shown to confer resistance to DOX $(4,8)$. The GS-X transport mechanism appears to be similar to the P-glycoprotein and its related transporters not only because of its ability to transport DOX with a relatively low $K_{\mathrm{m}}$ and its wide substrate specificity, but also because, similar to $\mathrm{P}$-glycoprotein, it is present in the canalicular membrane of hepatocytes (32). Thus, in a manner analogous to the P-glycoprotein, the GS-X conjugate transport mechanism may also function to ameliorate the cytotoxicity of DOX, daunomycin, vinblastine, and possibly other structurally unrelated amphiphilic compounds. Several malignant cell lines displaying the MDR phenotype have been described which appear to actively extrude DOX without expressing the P-glycoprotein (8-13). It is also interesting to note that a recent study (7) comparing the kinetics of DOX efflux between intact malignant cells with or without P-glycoprotein overexpression has shown that although the initial rate of DOX efflux was greater in malignant cells overexpressing the P-glycoprotein, the subsequent steady state rate of DOX efflux was equal in cells with or without P-glycoprotein expression (7), which suggests that xenobiotic transport mechanisms distinct from the P-glycoprotein may also play a role in mediating energy-dependent efflux of DOX. The results of present studies provide strong support to this contention.

The cytotoxicity of DOX towards drug-resistant malignant cells expressing the P-glycoprotein can be enhanced by multiple, structurally unrelated, relatively noncytotoxic compounds, presumably through competitive inhibition of the $P$ glycoprotein-mediated efflux of DOX (3-5). Because we have shown that GS-X conjugates are competitive inhibitors of DOX transport by this GS-X conjugate transporter, drugs such as ethacrynic acid which undergo extensive conjugation with GSH may also enhance the cytotoxicity of DOX. The coadministration of DOX with ethacrynic acid or other relatively nontoxic drugs which are metabolized to GS-X conjugates can thus be a novel approach for modulating the antineoplastic 
effects of DOX. Since the relatively high expression of glutathione $S$-transferases (GSTs) in many malignant tissues (33) may result in more rapid conversion of certain drugs (such as ethacrynic acid) to their GS-X conjugates, combinations of such drugs and DOX may be effective in selectively enhancing the cytotoxicity of DOX towards malignant cells overexpressing GSTs. This combination could be particularly useful for the treatment of drug-resistant malignancies overexpressing GST$\pi$ since ethacrynic acid is a preferred substrate of GST- $\pi$, the overexpression of which has been shown to decrease the efficacy of ethacrynic acid in enhancing the cytotoxicity of alkylating agents (34). Further studies are required to explore these possibilities for enhancing the cytotoxic effects of DOX in MDR phenotypes.

\section{Acknowledgments}

This study was supported in part by U.S. Public Health Service grant GM-32304 awarded by the National Institute for General Medicine Sciences to Y. C. Awasthi, and Veterans Administration Merit review to P. Zimniak. S. Awasthi thanks Dr. D. W. Powell, Chairman, Internal Medicine, University of Texas Medical Branch for research funds.

\section{References}

1. Chabner, B. A., and C. E. Myers. 1989. Clinical pharmacology of cancer chemotherapy. In Cancer Principles and Practice of Oncology. V. T. DeVita, S. Hellman, and S. A. Rosenberg, editors. J. B. Lippincott Co., Philadelphia. 349420.

2. Juliano, R. L., and V. Ling. 1976. A surface glycoprotein modulating drug permeability in Chinese hamster ovary cell mutants. Biochim. Biophys. Acta. 455:152-162.

3. Horio, M., M. M. Gottesman, and I. Pastan. 1988. ATP-dependent transport of vinblastine in vesicles from human multidrug-resistant cells. Proc. Natl. Acad. Sci. USA. 85:3580-3584.

4. Biedler, J. L. 1992. Genetic aspects of multidrug resistance. Cancer (Phila.). 70(Suppl. 6):1799-1809.

5. Nielson, D., and T. Skovsgaard. 1992. P-glycoprotein as multidrug transporter: a critical review of current multidrug resistant cell lines. Biochim. Biophys. Acta. 1139:169-183.

6. Spoelstra, E. C., H. V. Westerhoff, H. Dekker, and J. Lankelma. 1992. Kinetics of daunorubicin transport by P-glycoprotein of intact cancer cells. Eur. J. Biochem. 207:567-579.

7. Roepe, P. D. 1992. Analysis of the steady-state and initial rate of doxorubicin efflux from a series of multidrug-resistant cells expressing different levels of P-glycoprotein. Biochemistry. 31:12555-12564.

8. Roninson, I. B., J. E. Chin, K. Choi, P. Gros, D. E. Houseman, A. Fojo, D. W. Shen, M. M. Gottesman, and I. Pastan. 1986. Isolation of human mdr DNA sequences amplified in multidrug-resistant KB carcinoma cells. Proc. Natl. Acad. Sci. USA. 83:4583-4590.

9. Chen, Y. N., L. A. Mickley, A. M. Schwartz, E. M. Acton, J. Hwang, and A. T. Fojo. 1990. Characterization of adriamycin resistant human breast cancer cells which display over-expression of a novel resistance-related membrane protein. $J$. Biol. Chem. 265:10073-10080.

10. Slovak, M. L., G. A. Hoeltge, W. S. Dalton, and J. M. Trent. 1988. Pharmacologic and biologic evidence for differing mechanism of doxorubicin resistance in two human tumor cell lines. Cancer Res. 48:2793-2797.

11. Coley, H. M., P. Workman, and P. R. Twentyman. 1991. Retention of activity by selected anthracyclines in a multidrug resistant human large cell lung carcinoma line without P-glycoprotein hyperexpression. Br. J. Cancer. 63:351357.

12. Versantvoort, C. H. M., H. J. Broxterman, H. M. Pinedo, E. G. E. de Vries, N. Feller, C. M. Kuiper, and J. Lankelma. 1992. Energy-dependent processes involved in reduced drug accumulation in multidrug-resistant human lung cancer cell lines without P-glycoprotein expression. Cancer Res. 52:17-23.

13. Baas, F., A. P. M. Jongsma, H. J. Broxterman, R. J. Arecici, D. House- man, G. L. Scheffler, A. Riethorst, M. van Groenigen, A. W. M. Nieuwint, and H. Joenje. 1990. Non-P-glycoprotein mediated mechanisms for multidrug resistance precede P-glycoprotein expression during in-vitro selection for doxorubicin resistance in a human lung cancer cell line. Cancer Res. 50:5392-5398.

14. Srivastava, S. K., and E. Beutler. 1969. The transport of oxidized glutathione from human erythrocytes. J. Biol. Chem. 244:9-16.

15. Kondo, T., G. L. Dale, and E. Beutler. 1981. Studies on glutathione transport utilizing inside-out vesicles prepared from human erythrocytes. Biochim. Biophys. Acta. 645:132-136.

16. LaBelle, E. F., S. V. Singh, S. K. Srivastava, and Y. C. Awasthi. 1986. Dinitrophenyl glutathione efflux from human erythrocytes is a primary active ATP-dependent transport. Biochem. J. 238:443-449.

17. LaBelle, E. F., S. V. Singh, H. Ahmad, L. Wronski, S. K. Srivastava, and Y. C. Awasthi. 1988. A novel dinitrophenylglutathione-stimulated ATPase is present in human erythrocyte membranes. FEBS (Fed. Eur. Biochem. Soc.) Lett. 228:53-56.

18. Kondo, T., Y. Kawakani, N. Taniguchi, and E. Beutler. 1987. Glutathione disulfide-stimulated $\mathbf{M g}^{2+}$-ATPase of human erythrocyte membranes. Proc. Natl. Acad. Sci. USA. 84:7373-7377.

19. Sharma, R., S. Gupta, S. V. Singh, R. D. Medh, H. Ahmad, E. F. LaBelle, and Y. C. Awasthi. 1990. Purification and characterization of dinitrophenylglutathione ATPase of human erythrocytes and its expression in other tissues. Biochem. Biophys. Res. Commun. 171:155-161.

20. Singhal, S. S., R. Sharma, S. Gupta, H. Ahmad, P. Zimniak, A. Radominska, R. Lester, and Y. C. Awasthi. 1991. The anionic conjugates of bilirubin and bile salts stimulate ATP hydrolysis by $S$-(dinitrophenyl)glutathione ATPase of human erythrocyte. FEBS (Fed. Eur. Biochem. Soc.) Lett. 281:255-257.

21. Saxena, M., S. S. Singhal, S. Awasthi, S. V. Singh, E. F. LaBelle, P. Zimniak, and Y. C. Awasthi. 1992. Dinitrophenyl $S$-glutathione ATPase purified from human muscle catalyzes ATP hydrolysis in presence of leukotrienes. Arch. Biochem. Biophys. 298:231-237.

22. Awasthi, Y. C., H. S. Garg, D. D. Dao, C. A. Partridge, and S. K. Srivastava. 1981. Enzymatic conjugation of erythrocyte glutathione with 1-chloro-2,4dinitrobenzene: the fate of glutathione conjugate in erythrocytes and the effect of glutathione depletion on hemoglobin. Blood. 58:733-738.

23. Awasthi, S., S. K. Srivastava, F. Ahmad, H. Ahmad, and G. A. S. Ansari. 1993. Interactions of glutathione $S$-transferase $\pi$ with ethacrynic acid and its glutathione conjugate. Biochim. Biophys. Acta. 1164:173-178.

24. Minamide, L. S., and J. R. Bamburg. 1990. A filter paper dye-binding assay for quantitative determination of protein without interference from reducing agents or detergents. Anal. Biochem. 190:66-70.

25. Steck, T. L., and J. A. Kant. 1974. Preparation of impermeable ghosts and inside-out vesicles from human erythrocyte membranes. Methods Enzymol. 31A:172-180.

26. Dodge, J. T., C. Mitchell, and D. J. Hanahan. 1963. The preparation and chemical characteristics of hemoglobin-free ghosts of human erythrocytes. Arch. Biochem. Biophys. 100:119-130.

27. Ellman, G. L., K. D. Courtney, V. Andres, and R. M. Featherstone. 1961. A new and rapid colorimetric determination of acetylcholinesterase activity. Biochem. Pharmacol. 7:88-95.

28. Penefsky, H. S. 1977. Reversible binding of $P_{i}$ by beef heart mitochondrial adenosine triphosphatase. J. Biol. Chem. 252:2891-2899.

29. Awasthi, S., R. Sharma, Y. C. Awasthi, J. A. Belli, and E. P. Frenkel. 1992. The relationship of doxorubicin binding to membrane lipids with drug resistance. Cancer Lett. 63:109-116.

30. Benjamin, R. S. 1974. Pharmacokinetics of adriamycin (NSC-123127) in patients with sarcomas. Cancer Chemother. Rep. 58:271-273.

31. Keizer, H. G., G. J. Schuurhuid, H. J. Broxterman, J. Lankelma, W. G. E. J. Schoonen, J. van Rijn, H. M. Pinedoand, and H. Joenje. 1989. Correlation of multidrug resistance with decreased accumulation, altered subcellular drug distribution and increased P-glycoprotein expression in cultured SW-1573 human lung tumor cells. Cancer Res. 49:2988-2993.

32. Zimniak, P., S. A. Ziller, I. Panfil, A. Radominska, H. Wolters, F Kuipers, R. Sharma, M. Saxena, M. T. Moslen, M. Vore, et al. 1991. Identification of an anion-transport ATPase that catalyzed glutathione conjugate-dependent ATP hydrolysis in canalicular plasma membranes from normal rats and rats with conjugated hyperbilirubinemia (GY mutant). Arch. Biochem. Biophys. 292:534-538.

33. Tsuchida, S., and K. Sato. 1992. Glutathione transferases and cancer. Crit. Rev. Biochem. Mol. Biol. 27:337-384.

34. Kuzmich, S., L. A. Vanderveer, W. S. Walsh, F. P. LaCreta, and K. D. Tew. 1992. Increased levels of glutathione $S$-transferase- $\pi$ transcript as mechanism of resistance to ethacrynic acid. Biochem. J. 281:219-224. 